Historia y comunicación social

ISSN-e 1988-3056

\title{
El impacto de las fake news en la investigación en Ciencias Sociales. Revisión bibliográfica sistematizada
}

\author{
Ignacio Blanco Alfonso ${ }^{1}$; Carmen García Galera ${ }^{2}$; Santiago Tejedor Calvo ${ }^{3}$
}

Recibido: 30 de abril de 2019. / Aceptado: 23 de septiembre de 2019.

Resumen. Las noticias falsas o fake news vienen ocupando un papel protagonista a nivel político, social, mediático y también, científico. En este trabajo se desarrolla una revisión sistematizada de los estudios realizados hasta la fecha sobre las noticias falsas, partiendo de las principales bases de datos (Web of Science, Dialnet Plus) y con el fin de verificar qué temas han atraído la atención de la comunidad científica al respecto, qué técnicas de investigación se han utilizado en estos estudios, dónde se concentran geográficamente y qué campos quedan aún por cubrir en torno a este fenómeno. El estudio realizado refleja que aún queda camino por recorrer, en especial, en el ámbito iberoamericano que aparece menos representado dentro de la producción científica sobre las noticias falsas.

Palabras clave: Noticias falsas; revisión bibliográfica sistematizada; desinformación; Web of Science; Dialnet Plus

\section{[en] The impact of fake news on Social Science Research. Systematized bibliographic review}

\begin{abstract}
Fake news has begun to play a major role at the political, social, media, and even scientific level. In this work, a systematic review of the studies carried out to date on fake news has been developed starting from the main databases (Web of Science, Dialnet Plus), with the aim of verifying which topics have attracted the attention of the scientific community in this regard, what research techniques have been used in these studies, where these are geographically concentrated, and what fields still need to be investigated regarding this phenomenon. This study shows that there is still a long way to go, especially in the Ibero-American sphere that appears quantitatively less represented in the context of scientific production on fake news.
\end{abstract}

Keywords: Fake news; Systematized bibliographic review; disinformation; Web of Science; Dialnet Plus

Sumario: 1. Introducción; 2. ¿Por qué una revisión bibliográfica sistematizada?; 3. Metodología; 4. Resultados; 4.1 año y frecuencia de publicación; 4.2. Universidades o centros de investigación de los autores; 4.3. Países más destacados; 4.4. Áreas de conocimiento destacables; 4.5. Ranking de revistas con más artículos publicados; 4.6. Correlación temática entre descriptores clave; 4.7. Técnicas De Investigación; 5 . Conclusiones; 6 . Referencias bibliográficas.

\footnotetext{
1 Universidad CEU San Pablo.

iblanco@ceu.es

2 Universidad Rey Juan Carlos.

carmen.garcia@urjc.es

3 Universidad Autónoma de Barcelona. santiago.tejedor@uab.cat
} 
Cómo citar: Blanco Alfonso, I.; García Galera, C.; Tejedor Calvo, S. (2019). El impacto de las fake news en la investigación en Ciencias Sociales. Revisión bibliográfica sistematizada, en Historia y comunicación social 24 (2), 449-469.

\section{Introducción}

En el último debate televisivo entre los candidatos a la Presidencia del Gobierno en España se contó por primera vez con diferentes equipos de verificación que desvelaban las afirmaciones falsas de los candidatos durante el debate (RTVE, 2019; eldiario.es, 2019). Este hecho podría considerarse como un efecto de esa incertidumbre social y global que han traído consigo las fake news. Si se presta atención a la última década, los temas a los que se alude cuando se habla de las fake news han girado hacia el terreno político debido, en parte, al alto número de procesos electorales -y la controversia generada en torno a ellos o a los candidatos o propuestas presentadasque han tenido lugar en países como Francia, Reino Unido, Alemania, España y, en especial, Estados Unidos (Lowrey, 2017 citado en Vizoso y Vazquez, 2019). Tal es la repercusión que está teniendo este término que incluso, el Collins Dictionary eligió el término de fake news como la palabra del año en 2017, tras un proceso marcado por el éxito de programas satíricos que contribuyeron a la eclosión de dicha palabra en el dominio público (Love, 2007; Tally, 2011).

Por otra parte, cabe mencionar que el fenómeno de la fake news conecta con el gran problema filosófico de la percepción de la realidad. Del mismo modo que los prisioneros de la caverna de Platón (Libro VII de La República) confundían las sombras de los objetos con los objetos mismos, así el hombre contemporáneo parece confundir de los hechos proyectados en las pantallas con los hechos reales.

Este problema de raíz filosófica podría representar una amenaza política porque las democracias contemporáneas se basan en la existencia de una ciudadanía libre y bien informada, capaz de tomar decisiones complejas en contextos políticos polarizados (La Vanguardia, 2019). Sin embargo, no existe libertad de elección sin información veraz, de ahí que las fake news trasciendan el plano filosófico para erigirse en una gran amenaza política y social. El nuevo ecosistema mediático, caracterizado por ser un contexto de desorden informativo (Wardle \& Derakhshan, 2017), ha contribuido a la proliferación de este tipo de contenidos (Jan \& Kim, 2018) al tiempo que ha consolidado un escenario muy proclive a la generación de noticas falsas (Ireland, 2018; Southwell et al., 2018).

Casi a comienzos del pasado siglo, Walter Lippmann se anticipó a nuestro tiempo al escribir que, en sentido estricto, la crisis actual de la democracia es una crisis de su periodismo (Blanco, 2011). Los críticos de la sociedad de masas advirtieron que la democracia no sería posible sin la concurrencia de una prensa robusta y rigurosa. Una vez superado el convulso siglo XX, volvemos al punto de partida de la contemporaneidad al haberse instalado en la política actual el paradigma de que la mentira es el mensaje (Gessen, 2016). Es la llamada posverdad, un clima social moldeado por la acción de la propaganda, en el que los razonamientos construidos sobre los hechos verificados (logos) son arrasados por mensajes simbólicos que apelan a emociones primarias del ser humano (pathos), mermando el análisis racional y provocando respuestas emocionales. En este contexto triunfan las llamadas noticias falsas 
o fake news, contenidos producidos con apariencia de informaciones periodísticas que, sin embargo, ni se procesan ni se distribuyen por los cauces convencionales del periodismo.

A pesar de que noticia falsa es un oxímoron problemático, se van conociendo algunos elementos comunes a estos contenidos: tienen intención perniciosa, habitualmente de raíz política o económica; la temática es transversal (están documentadas fake news en el terreno de la política, la medicina, la economía, la historia...); suelen apelar a supuestas noticias llamativas, impactantes o alarmantes, lo que favorece que se propaguen un $10 \%$ más que las noticias auténticas, y que lo hagan con gran virulencia y rapidez (Vosoughi, Roy y Aral, 2018); el impacto social es intergeneracional, aunque afectan substancialmente a públicos vulnerables como los mayores de 65 años, que se muestran siete veces más proclives que los jóvenes a compartirlas (Guess, Nagler y Tucker, 2019); la apariencia es cada vez más sofisticada: la última generación son las llamadas deep fake news, contenidos audiovisuales en los que un personaje público dice cosas que nunca dijo o hace cosas que nunca hizo. Son creados por una inteligencia artificial y su montaje es prácticamente indetectable por el ojo humano.

A pesar de que las acciones de contención comenzaron en 2015 (por ejemplo, la EU East StratCom Task Force, impulsada por el Consejo Europeo), el Eurobarómetro de abril de 2018 indica que el $37 \%$ de los europeos recibe noticias falsas diariamente, y el $31 \%$, una vez a la semana. El 83\% de los europeos piensa, además, que la desinformación es un grave problema para la estabilidad democrática. De acuerdo con las previsiones de la consultora Gartner, en 2022 los ciudadanos occidentales consumirán más noticias falsas que auténticas.

\section{2. ¿Por qué una revisión bibliográfica sistematizada?}

La realización de revisiones de la literatura científica permite tener un conocimiento actualizado sobre un determinado tema, además de proporcionar a la comunidad científica nuevas líneas de investigación aún por explorar en ese campo de estudio. De hecho, el saber acumulado es la base sobre la que se desarrollan las nuevas investigaciones mediante un proceso de refutación, confirmación o la exploración de nuevas formulaciones que contribuyan a la explicación de los fenómenos de estudio (Guirao, 2015)

En este contexto, se abren paso las denominadas revisiones sistematizadas, revisiones bibliográficas destinadas a una variedad de objetivos, uno de los principales es la elaboración de estados de la cuestión en cualquier ámbito de las Ciencias Humanas y Sociales. Las revisiones sistematizadas proporcionan un marco de trabajo riguroso y sistemático (de aquí su nombre) para llevar a cabo revisiones bibliográficas (Codina, 2018).

Así pues, una revisión sistematizada es una revisión de la bibliografía, si bien estas revisiones no son solamente una revisión de la literatura (Wilson, 2013). En la actualidad, la producción científica en muy diferentes ámbitos ha experimentado un crecimiento exponencial, incremento que no solo responde al derecho de la sociedad a conocer sino también a la obligación de los investigadores a difundir.

Si bien tradicionalmente la búsqueda bibliográfica suponía para el investigador horas de recopilación documental presencial en bibliotecas y centros de estudio, la 
tecnología también ha facilitado un cambio importante en este aspecto, en tanto en cuanto, las bases de datos han dado paso a una revisión de la literatura de manera sistemática y fiable, de forma virtual.

Una base de datos es una colección sistemáticamente estructurada de datos e informaciones. En el caso de las bases de datos académicas, estos datos e informaciones consisten en documentos producidos como resultado de estudios e investigaciones, y adquieren la forma de artículos publicados en revistas científicas y de actas de congresos (Codina, 2017).

Con relación al fenómeno de las fake news, autores como Wardle y Derakhshan (2017), entre otros, aluden a la necesidad de desarrollar estudios que delimiten conceptualmente el alcance del término y la globalidad de la problemática que este suscita, especialmente, en el actual panorama mediático (Tornero y Varis, 2010; Middaugh, 2019). Este aspecto incide en la pertinencia de trabajos diagnósticos y descriptivos sobre el volumen y enfoque de los estudios existentes en este campo.

A ello se unen los resultados de diferentes trabajos como los de Hargittai et al. (2010), Wineburg y McGrew (2017) o Tornero et al. (2018), entre otros, que subrayan la necesidad de potenciar la media literacy entre el público infantil y juvenil frente al fenómeno de las noticias falsas. Las investigaciones de Tannen (1999), Thompson (2016) y Sherry Turkle (2015) han aludido a la indisociable relación entre la eclosión de las noticias falseadas y el actual deterioro de la esfera pública, política y mediática. En este contexto, estudios como los de Fletcher et al. (2018), Musgrove et al. (2018) o Vosoughi (2018) han advertido que las plataformas de noticias falsas poseen mayor grado de interacción que medios consolidados o que las personas superan a los bots en la difusión de este tipo de contenidos (conlicencia.com, 2017). Se plantea, en definitiva, la necesidad de una investigación de cariz descriptivo que analice, estructure y describa el conjunto de estudios y trabajos científicos desarrollados en este ámbito. En esta línea, el presente artículo propone una revisión sistematizada de la literatura científica que existe con relación al fenómeno de las fake news.

\section{Metodología}

El objetivo general de esta investigación es realizar una revisión bibliográfica sistematizada descriptiva sobre noticias falsas o fake news, y ofrecer un panorama del estado del arte a partir de la producción científica nacional e internacional en los últimos años. Este tipo de revisiones se constituyen como una herramienta esencial para sintetizar la información sobre un campo en particular, registrar los pasos asumidos durante la investigación en el campo específico de interés e iniciar futuras líneas de investigación en aquellas áreas que todavía no han sido exploradas (Boettla y Gambara, 2006b; Ferreira, Uurrútia y Alonso-Coello, 2011, citado en Camilli, 2018).

La hipótesis de esta investigación es que la principal producción científica sobre fake news procede del ámbito científico anglosajón, se centra en el campo de la comunicación y se concentra en los dos últimos años, fruto de los acontecimientos políticos que tuvieron lugar en el año 2016 y siguientes.

El trabajo que aquí se presenta es, además, una revisión descriptiva ya que centra su atención en revelar patrones existentes en la literatura, lo que requiere cierta cuantificación que se evidencia en un análisis de frecuencia a través del número de publicaciones, diseño metodológico de las investigaciones y resultados. Se asume 
cada estudio como un dato en sí mismo y se identifican las tendencias y los patrones entre los documentos seleccionados. El resultado de tal revisión, a menudo, es representativo del estado del arte en el dominio investigado (Camilli, 2018).

Las preguntas de investigación, pues, se centran en conocer si estamos ante un fenómeno de gran impacto entre la comunidad científica de Ciencias Sociales, hasta qué punto es novedoso, y cuáles han sido los principales objetos de estudio, grupos de interés y metodología de investigación en torno a las noticias falsas.

Desde el punto de vista de la sistemática de esta investigación, se partió en primer lugar de la elaboración de un protocolo para la revisión y organización de la literatura, tanto en inglés como en español. Así, siguiendo a Codina (2018) y su planteamiento sobre las revisiones bibliográficas, el primer paso consistió en establecer claramente las fases de trabajo.

La búsqueda, como primera fase de esta revisión, se realizó en varias etapas. La primera consistió en una exploración tentativa para establecer qué descriptores se tenían que tener en cuenta a la hora de componer la matriz final. Esta búsqueda se realizó a partir de los términos "fake news" y "noticias falsas" entre los artículos más citados en la Web of Science, en Scopus, y en el portal bibliográfico Dialnet Plus, esta última con el fin de detectar contenidos científicos iberoamericanos de calidad, pero no incluidos en las colecciones de las otras dos bases de datos.

En el caso de la Web of Science y de Scopus, observamos una coincidencia casi total entre los artículos resultantes. El término "fake news" estaba presente en el $50 \%$ de las palabras clave de los 10 artículos más citados de la Web of Science, y el $70 \%$ de los artículos más citados de Scopus. Esta primera búsqueda arrojó también otros descriptores como "misinformation", "disinformation", "news verification", "false news" y "fact checking", que utilizaríamos posteriormente en el análisis de resultados. Decidimos descartar los artículos de Scopus y centrar nuestro análisis en los resultados obtenidos de la base de datos Social Science Citation Index (SSCI) con los filtros "solo artículos" y el descriptor "fake news" en título, abstract y keywords.

En el caso de Dialnet Plus, introdujimos los siguientes criterios de búsqueda conducentes a obtener "solo artículos", "en español", que hubiesen sido publicados en revistas indizadas en Latindex, y clasificadas en CIRC A y CIRC B de Ciencias Sociales. Esta búsqueda arrojó un total de 12 artículos, 8 de los cuales recogían como palabra clave "fake news" o "noticias falsas". Otros conceptos como "posverdad" aparecían en cinco de los artículos, o "desinformación" solo en uno.

A continuación, procedimos a depurar las coincidencias de los artículos que habían aparecido en SSCI y en Dialnet Plus, y alcanzamos a confeccionar el banco de documentos (Codina, 2018), que contenía dos matrices de análisis (ver Tabla 01): una para los artículos alojados en SSCI de la Web of Science, con un total de 164 ítems (N1 = 164); y otra para los artículos indizados en Dialnet Plus, con un total de 8 artículos $(\mathrm{N} 2=8)$. 


\begin{tabular}{|l|c|c|c|}
\hline \multicolumn{1}{|c|}{ Base de datos } & \multicolumn{1}{|c|}{ Criterios de búsqueda } & $\begin{array}{c}\text { N. de } \\
\text { artículos }\end{array}$ & $\begin{array}{c}\text { Id de la } \\
\text { matriz }\end{array}$ \\
\hline $\begin{array}{l}\text { Web of Science Social } \\
\text { Science Citation Index } \\
\text { (SSCI) }\end{array}$ & $\mathrm{TS}=$ "fake news" + "Solo artículos" & 164 & $\mathrm{~N} 1$ \\
\hline Dialnet Plus & $\begin{array}{l}\text { "Fake news" + "Noticias falsas" + Solo } \\
\text { artículos + Revistas Latindex + Revistas } \\
\text { CIRC A + B de Ciencias Sociales }\end{array}$ & 8 & N2 \\
\hline
\end{tabular}

Tabla 01. Banco de documentos analizados.

Elaboración propia.

A partir de aquí, tomamos en consideración los siguientes criterios para efectuar nuestro análisis (Camilli, 2018; López et al, 2019): año de publicación (del que obtendríamos la frecuencia de publicación), centros/universidades de filiación de los autores de los artículos; países de publicación de los artículos; área de conocimiento de los artículos dentro de las Ciencias Sociales; listado de revistas con más artículos publicados sobre fake news; correlación temática entre descriptores clave; y técnicas de investigación (ver Tabla 02). Una vez concluida la codificación, y antes de comenzar con la fase de interpretación y síntesis de los resultados encontrados, se realizaron diferentes pruebas entre codificadores para asegurar la fiabilidad y validez del trabajo realizado (Sánchez, 2005; Peña, 2012).

\begin{tabular}{|c|l|}
\hline Criterio & \multicolumn{1}{c|}{ Denominación } \\
\hline 1 & Año de publicación / frecuencia de publicación \\
\hline 2 & Centros/universidades de filiación de los autores \\
\hline 3 & Países de publicación de los artículos \\
\hline 4 & Área de conocimiento de los artículos \\
\hline 5 & Ranking de las revistas con más artículos sobre fake news \\
\hline 6 & Correlación temática entre descriptores clave \\
\hline 7 & Técnicas de investigación \\
\hline
\end{tabular}

Tabla 02. Criterios para el análisis.

Elaboración propia.

\section{Resultados}

Los resultados se presentan por este orden: criterio de análisis ordenado del 1 al 7; dentro de cada criterio, primero se exponen los resultados de la matriz N1 (SSCI) WoS) y a continuación, los resultados de la matriz N2 (Dialnet Plus).

\subsection{Año y frecuencia de publicación}

En el caso de la matriz N1, encontramos que los primeros artículos indexados en el índice SSCI aparecieron en 2005 y 2006. Si bien el artículo de 2005 (un estudio de caso del popular programa estadounidense "The Daily Show", presentado por John 
Stewart y emitido por el canal Comedy Central) analizaba las fake news como mensajes satíricos enmarcados en la línea humorística del programa, el artículo de 2006 denunciaba el uso de imágenes falsas en las retransmisiones televisivas de la $\mathrm{ABC}$ sobre la guerra de Bosnia. Por lo tanto, se trata de un análisis pionero directamente conectado con el sentido actual de las fake news.

Como se puede ver en el gráfico 1, desde aquellas fechas, la frecuencia de publicación de investigaciones sobre las noticias falsas se mantiene por debajo del 1\% del total del corpus analizado. Sin embargo, en 2013 ven la luz 5 artículos (3\% de la muestra), entre los que destacan 4 análisis en los que se relaciona la expresión fake news con la sátira política: "The Impact of Real News about "Fake news": Intertextual Processes and Political Satire"; "Developing a Normative Approach to Political Satire: A Critical Perspective"; "Trusted puppets, tarnished politicians: Humor and cynicism in Berlusconi's Italy", y "From Critique to Mobilization: The Yes Men and the Utopian Politics of Satirical Fake news".

Junto al análisis de la sátira política, también en 2013 encontramos un artículo que ya relaciona las fake news con la viralización de contenidos a través de Facebook. En la investigación experimental titulada "The anatomy of the virus-marketing campaign of book titled "A vilaghalo metaforai"”, el autor describe cómo ha conseguido viralizar un mensaje en Facebook sin necesidad de mediación profesional, tan solo con una pequeña aportación económica y a través de una reducida comunidad de usuarios; ya se empezaba a marcar el camino que iban a seguir los emisores de noticias falsas poco tiempo después.

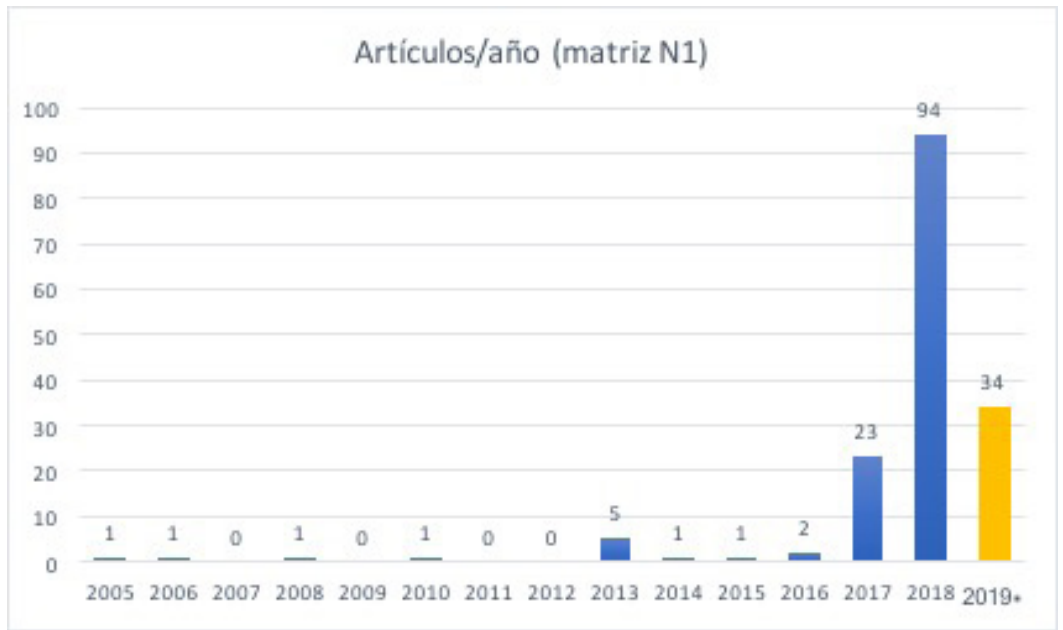

Gráfico 01. Artículos por año (matriz N1).

Elaboración propia con datos de SSCI-WoS

* enero-abril de 2019. La expectativa de crecimiento es de 102 artículos cuando termine este año.

Como se puede observar en el gráfico 1, tras las campañas políticas del referéndum del Brexit (junio 2016) y de las elecciones presidenciales en Estados Unidos (noviembre 2016) se dispara el número de investigaciones académicas sobre el fenómeno de las fake news. En 2017 ven la luz 23 artículos (el 14\% del corpus analizado), y en 2018, 94 (el 57,3\% del corpus). El hecho de que en 2018 se publi- 
quen cuatro veces más artículos que en 2017 se explica por el tiempo de realización de las investigaciones y por la cadencia de publicación de las revistas arbitradas. Como se puede observar en este mismo gráfico 1, los 34 artículos (20,7\% del corpus) publicados en lo que va de 2019 generan una expectativa de crecimiento de hasta 102 contribuciones. Esta línea ascendente de investigaciones pone de manifiesto el creciente interés de la comunidad científica por el tema. Del corpus de 164 artículos analizados, el "Brexit" está presente (en título, abstract y/o keywords) en 4 artículos publicados entre 2017 y 2019 (ver Tabla 03):

\begin{tabular}{|l|c|}
\hline \multicolumn{1}{|c|}{ TS= "Brexit" } & $\begin{array}{c}\text { Año de } \\
\text { publicación }\end{array}$ \\
\hline The Brexit Botnet and User-Generated Hyperpartisan News & 2019 \\
\hline $\begin{array}{l}\text { The disinformation order: Disruptive communication and the decline of } \\
\text { democratic institutions }\end{array}$ & 2018 \\
\hline $\begin{array}{l}\text { Back to the future. How UK-based news organisations are rediscovering } \\
\text { objectivity }\end{array}$ & 2018 \\
\hline $\begin{array}{l}\text { The Rise of Post-truth Populism in Pluralist Liberal Democracies: Chal- } \\
\text { lenges for Health Policy }\end{array}$ & 2017 \\
\hline
\end{tabular}

Tabla 03. Artículos relativos al Brexit.

Elaboración propia con datos de SSCI-WoS

Igualmente, de los 164 artículos analizados, "Donald Trump" está presente (en título, abstract y/o keywords) en 13 artículos publicados entre 2017 y 2019:

\begin{tabular}{|l|c|}
\hline \multicolumn{1}{|c|}{ TS= "Donald Trump" } & $\begin{array}{c}\text { Año de } \\
\text { publicación }\end{array}$ \\
\hline Psychological Underpinnings of Post-Truth in Political Beliefs & 2019 \\
\hline $\begin{array}{l}\text { What the fake? Assessing the extent of networked political spamming and } \\
\text { bots in the propagation of \#fakenews on Twitter }\end{array}$ & 2019 \\
\hline $\begin{array}{l}\text { Trump vs. media. Treatment of the press from the US president's Twitter } \\
\text { account }\end{array}$ & 2018 \\
\hline $\begin{array}{l}\text { Fake news or Weak Science? Visibility and Characterization of Antivaccine } \\
\text { Webpages Returned by Google in Different Languages and Countries }\end{array}$ & 2018 \\
\hline $\begin{array}{l}\text { Fabricating the American Dream in US media portrayals of Syrian refu- } \\
\text { gees: A discourse analytical study }\end{array}$ & 2018 \\
\hline $\begin{array}{l}\text { The disinformation order: Disruptive communication and the decline of } \\
\text { democratic institutions }\end{array}$ & 2018 \\
\hline $\begin{array}{l}\text { The Self-Radicalization of White Men: "Fake news" and the Affective Ne- } \\
\text { tworking of Paranoia }\end{array}$ & 2018 \\
\hline $\begin{array}{l}\text { Fake news as a floating signifier: hegemony, antagonism and the politics } \\
\text { of falsehood }\end{array}$ & 2018 \\
\hline $\begin{array}{l}\text { Citizen curation in online discussions of Donald Trump's Presidency. Sha- } \\
\text { ring the News on Mumsnet }\end{array}$ & 2018 \\
\hline $\begin{array}{l}\text { (Re)conceptualizing digital literacies before and after the election of } \\
\text { Trump (2018) }\end{array}$ & 2018 \\
\hline
\end{tabular}




\begin{tabular}{|l|c|}
\hline The media coverage of fake news, a hype? & 2018 \\
\hline $\begin{array}{l}\text { Hacking the Research Library: Wikipedia, Trump, and Information Litera- } \\
\text { cy in the Escape Room at Fresno State }\end{array}$ & 2017 \\
\hline The hollow man Donald Trump, populism, and post-truth politics & 2017 \\
\hline
\end{tabular}

Tabla 04. Artículos relativos a Donald Trump.

Elaboración propia con datos de SSCI-WoS

En el caso de la matriz N2, los ocho artículos rescatados de Dialnet Plus presentan la siguiente cronología:

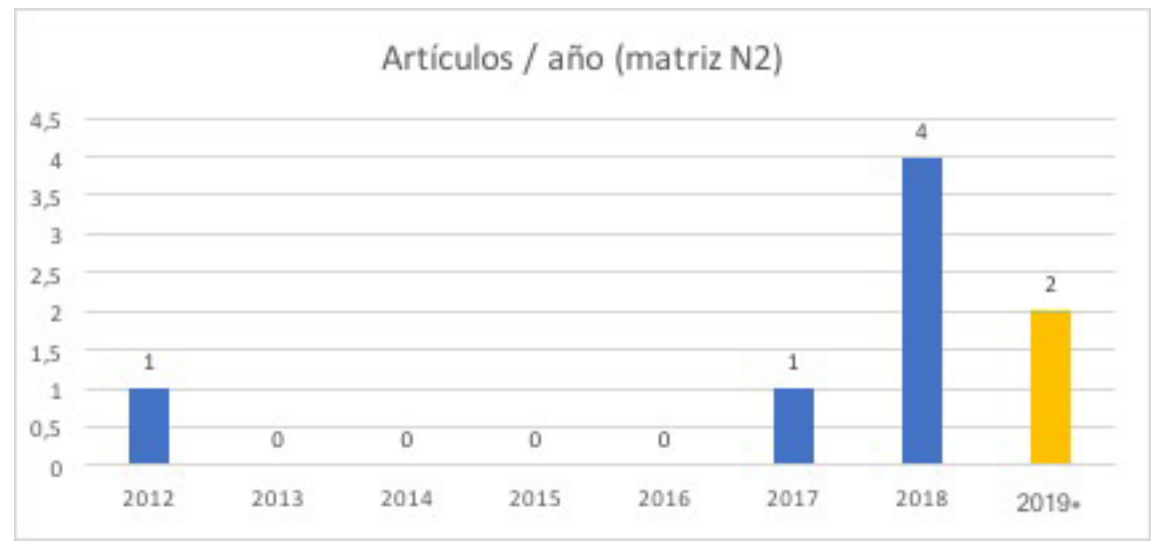

Gráfico 02. Artículos por año (matriz N3)

Elaboración propia con datos de Dialnet Plus

* enero-abril de 2019. La expectativa de crecimiento es de 6 artículos cuando termine este año.

El análisis de este gráfico 2 es similar al de la matriz N1, aunque la diferencia en el número de artículos sea significativa. Se observa la misma frecuencia de publicación, con un artículo solitario en 2012 y un notable incremento a partir de 2018, lo que podría justificarse, al igual que en el caso anterior, en base a la irrupción de las noticias falsas en el ecosistema político democrático tras experiencias como la del Brexit o las elecciones presidenciales de Estados Unidos.

\subsection{Universidades o centros de investigación de los autores}

Dado que la matriz N1 está compuesta por 164 artículos, en este criterio hemos seleccionado solo las universidades que concentran 3 o más artículos. El resultado se puede observar en el gráfico 3: 


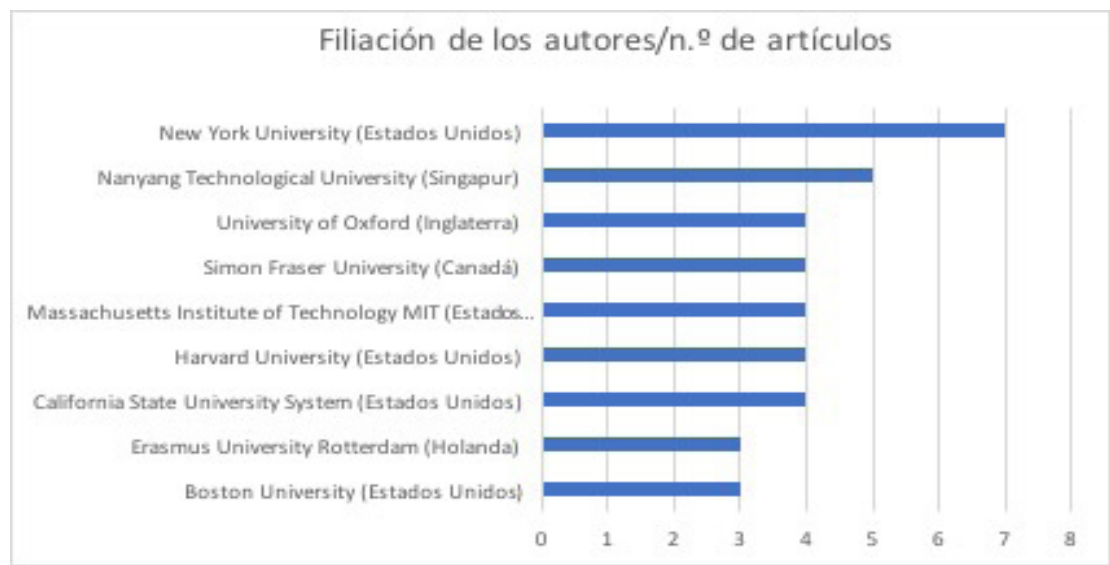

Gráfico 03. Filiación de los autores / $\mathrm{N}^{\mathrm{o}}$ de artículos Elaboración propia con datos de SSCI-WoS

Este mapa universitario muestra la preponderancia de centros de investigación estadounidenses: entre las 10 Universidades que han publicado más de 3 artículos, 5 son de Estados Unidos, 2 de Singapur, 2 europeas y 1 de Canadá. En el caso de las filiaciones de los autores de los 8 artículos de la matriz N2, como el número es reducido ofrecemos todos los datos ordenados en la siguiente tabla:

\begin{tabular}{|l|l|l|}
\hline \multicolumn{1}{|c|}{ Título del artículo } & \multicolumn{1}{|c|}{ Autor/es } & \multicolumn{1}{c|}{ Filiación del autor/es } \\
\hline $\begin{array}{l}\text { La información digital enjuiciada } \\
\text { por la prensa }\end{array}$ & Raymond Colle & $\begin{array}{l}\text { Asociación chilena de inves- } \\
\text { tigadores de comunicación }\end{array}$ \\
\hline $\begin{array}{l}\text { Fact-Checking en los debates elec- } \\
\text { torales televisados de las elecciones } \\
\text { generales de 2015 y 2016 }\end{array}$ & $\begin{array}{l}\text { Andrés Mazaira Cas- } \\
\text { tro, José Rúas Araújo } \\
\text { e Iván Puentes Rivera }\end{array}$ & $\begin{array}{l}\text { Universidad de Vigo y Uni- } \\
\text { versidad de A Coruña }\end{array}$ \\
\hline $\begin{array}{l}\text { Noticias falsas y libertad de expre- } \\
\text { sión e información. El control de los } \\
\text { contenidos informativos en la red }\end{array}$ & Cristina Pauner Chulvi & Universitat Jaume I \\
\hline $\begin{array}{l}\text { ¿Cómo afrontar las noticias falsea- } \\
\text { das mediante la alfabetización pe- } \\
\text { riodística? Estado de la cuestión }\end{array}$ & $\begin{array}{l}\text { José Manuel Pérez } \\
\text { Tornero, Samy Tayie, } \\
\text { Cristina Pulido }\end{array}$ & $\begin{array}{l}\text { Universidad Autónoma de } \\
\text { Barcelona }\end{array}$ \\
\hline $\begin{array}{l}\text { WhatsApp como herramienta de } \\
\text { verificación de fake news. El caso } \\
\text { de B de Bulo }\end{array}$ & $\begin{array}{l}\text { María Bella Palomo } \\
\text { Torres y Jon A. Seda- } \\
\text { no Amundarain }\end{array}$ & Universidad de Málaga \\
\hline $\begin{array}{l}\text { Estratagemas de la posverdad } \\
\text { Pilar Carrera Álvarez }\end{array}$ & $\begin{array}{l}\text { Universidad Carlos III de } \\
\text { Madrid }\end{array}$ \\
\hline $\begin{array}{l}\text { El Slow Journalism en la era de la } \\
\text { "infoxicación" }\end{array}$ & $\begin{array}{l}\text { Samia Benaissa Pe- } \\
\text { driza }\end{array}$ & $\begin{array}{l}\text { Universidad Complutense } \\
\text { de Madrid }\end{array}$ \\
\hline $\begin{array}{l}\text { El rumor del nopal chino: construc- } \\
\text { ción institucional y efectos sociales } \\
\text { de noticias falsas }\end{array}$ & $\begin{array}{l}\text { Bruno Lutz y Alexan- } \\
\text { der Padilla }\end{array}$ & $\begin{array}{l}\text { Universidad Autónoma Me- } \\
\text { tropolitana-Xochimilco }\end{array}$ \\
\hline
\end{tabular}

Tabla 04. Filiaciones de los artículos (matriz N2).

Elaboración propia con datos de Dialnet Plus 
En esta matriz observamos que 6 de los 8 artículos han sido publicados en centros de investigación o universidades españolas, mientras que solo 2 artículos están firmados por autores de países de Latinoamérica, uno de México y otro de Chile.

\subsection{Países más destacados}

Estados Unidos (79 artículos) se sitúa a la cabeza -y con gran diferencia respecto al resto de países- en cuanto a investigación acerca de las fake news (Gráfico 4) se refiere. Le sigue a cierta distancia Reino Unido, que aporta 17 artículos, Australia 13 y Canadá, 11.

España, con 9 artículos, ocupa un interesante quinto lugar en este escalafón por delante de países como Holanda (8), Singapur (6), Suecia (5), Alemania (4), Noruega (4), República Popular de China (4) o Corea del Sur (4), como se aprecia en el gráfico 4 .

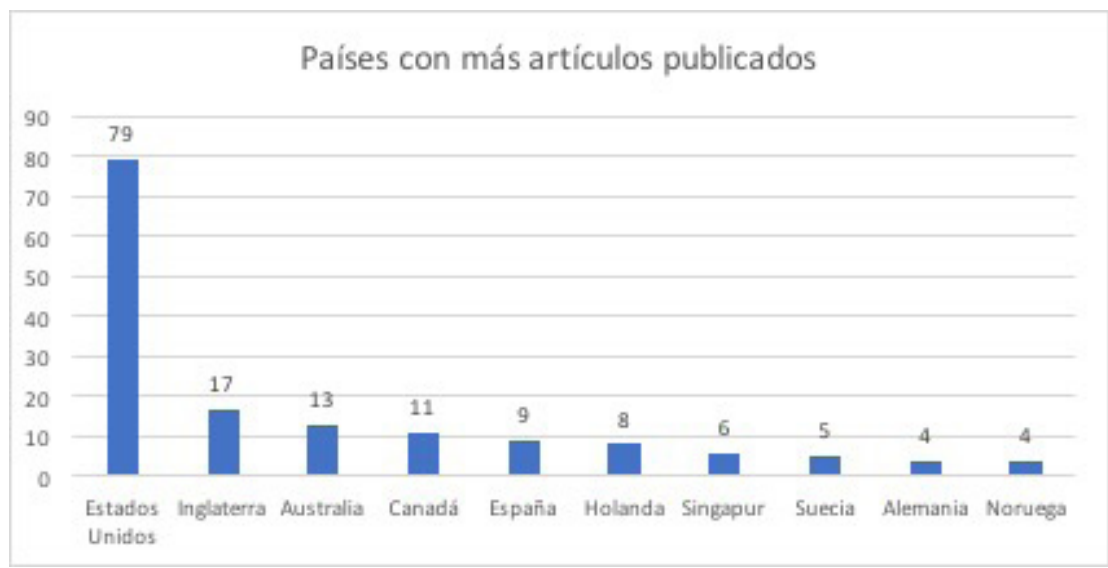

Gráfico 4. Artículos por países.

Elaboración propia con datos de SSCI-WoS

Obviamente, este mapa geográfico se refleja en el idioma de publicación de los 164 artículos que conforman el corpus: 150 en inglés; 6 en español; 2 en holandés; 2 en húngaro; 1 en afrikáans; 1 en checo; 1 en alemán; 1 en ruso.

\section{4. Áreas de conocimiento destacables}

Como se explicó en la metodología, el corpus de análisis procede del Social Science Citation Index, lo que supone de antemano un filtro temático que excluye todo lo que no esté catalogado como Ciencias Sociales. Hecha esta salvedad, en el gráfico 5 se observa un claro dominio de las aproximaciones al fenómeno de las fake news desde el campo de la Comunicación, que representan el 32,9\% del total (54 artículos). Esto puede responder a que la Comunicación es el ámbito de las Ciencias Sociales donde con mayor preocupación se percibe la amenaza de la desinformación y la manipulación de las noticias (ver gráfico 5). 


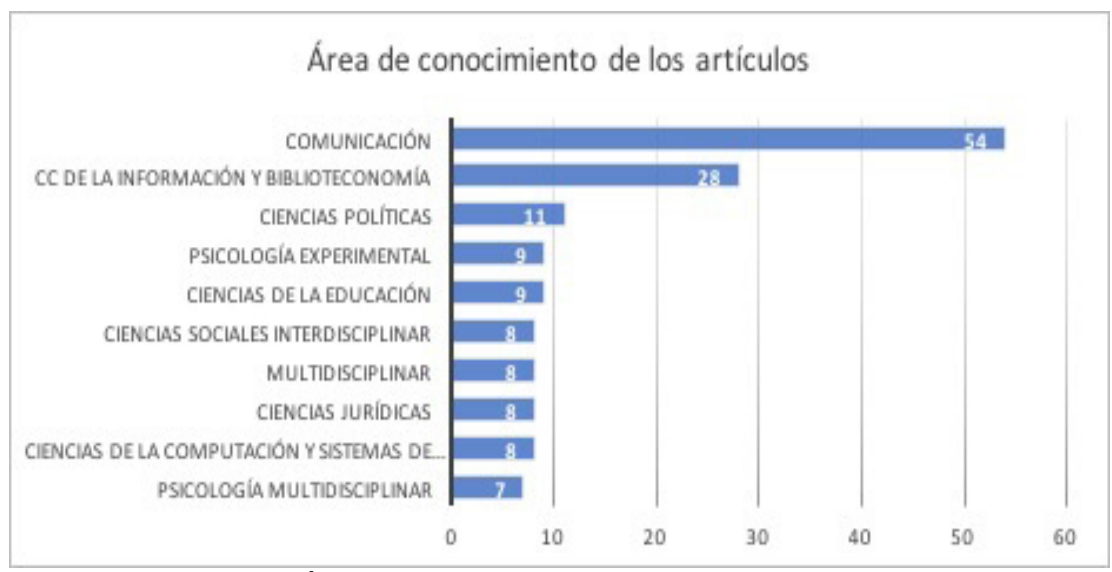

Gráfico 5. Área de conocimiento de los artículos.

Elaboración propia con datos de SSCI-WoS

En este mismo gráfico, se puede observar que las investigaciones efectuadas desde la Biblioteconomía y Ciencias de la Información representan el 17\% del total (28 artículos). La suma de ambos campos temáticos representa casi el 50\% del total de investigaciones sobre fake news (82 artículos), lo que decanta claramente el volumen de producción científica hacia las áreas de la Comunicación y la Información, que están liderando la investigación de este fenómeno.

Muy por detrás aparecen otras disciplinas en Ciencias Sociales, lo que viene a sugerir que las eventuales soluciones para el fenómeno de las noticias falsas procederán, principalmente, de investigaciones realizadas en Comunicación.

\subsection{Ranking de revistas con más artículos publicados}

Entre las revistas de la matriz N1 que han publicado más artículos sobre las fake news, cabe destacar las siguientes:

\begin{tabular}{|c|c|c|c|c|}
\hline $\begin{array}{l}\text { Nombre de la } \\
\text { revista }\end{array}$ & Campo temático & Editor & $\begin{array}{c}\text { Factor de } \\
\text { impacto y } \\
\text { posición }\end{array}$ & $\begin{array}{l}\text { N. }{ }^{\circ} \text { de } \\
\text { artículos }\end{array}$ \\
\hline $\begin{array}{l}\text { El profesional } \\
\text { de la informa- } \\
\text { ción }\end{array}$ & $\begin{array}{l}\text { Revista especializada en comu- } \\
\text { nicación, información, biblioteca } \\
\text { y tecnologías de la información }\end{array}$ & $\begin{array}{l}\text { El profe- } \\
\text { sional de la } \\
\text { información } \\
\end{array}$ & \begin{tabular}{|l|} 
JCR Q2 \\
$(43$ de 88$)$ \\
$F i=1,318$ \\
\end{tabular} & 7 \\
\hline $\begin{array}{l}\text { Digital } \\
\text { Journalism }\end{array}$ & $\begin{array}{l}\text { Revista especializada en la } \\
\text { transformación digital del perio- } \\
\text { dismo }\end{array}$ & $\begin{array}{l}\text { Taylor \& } \\
\text { Francis }\end{array}$ & -- & 6 \\
\hline $\begin{array}{l}\text { Journalism } \\
\text { Practice }\end{array}$ & $\begin{array}{l}\text { Revista especializa en la práctica } \\
\text { profesional del periodismo }\end{array}$ & $\begin{array}{l}\text { Taylor \& } \\
\text { Francis }\end{array}$ & \begin{tabular}{|l|} 
JCR Q2 \\
$(29$ de 84$)$ \\
$F i=1,678$ \\
\end{tabular} & 6 \\
\hline $\begin{array}{l}\text { Computers } \\
\text { in Human } \\
\text { Behavior }\end{array}$ & $\begin{array}{l}\text { Revista especializada en el estu- } \\
\text { dio de las computadoras desde } \\
\text { una perspectiva psicológica }\end{array}$ & Elsevier & \begin{tabular}{|l|} 
JCR Q1 \\
$(8$ de 85$)$ \\
$F i=3,536$ \\
\end{tabular} & 5 \\
\hline
\end{tabular}




\begin{tabular}{|l|l|l|l|l|}
\hline $\begin{array}{l}\text { Journalism } \\
\text { Studies }\end{array}$ & $\begin{array}{l}\text { Revista especializada en el cam- } \\
\text { po del periodismo }\end{array}$ & $\begin{array}{l}\text { Taylor \& } \\
\text { Francis }\end{array}$ & $\begin{array}{l}\text { JCR Q2 } \\
(25 \text { de 84 }) \\
F i=1,735\end{array}$ & 5 \\
\hline $\begin{array}{l}\text { New Media \& } \\
\text { Society }\end{array}$ & $\begin{array}{l}\text { Revista interdisciplinar centrada } \\
\text { en la transformación social y } \\
\text { tecnológica de los medios de } \\
\text { comunicación }\end{array}$ & SAGE & $\begin{array}{l}\text { JCR Q1 } \\
(4 \text { de 84) } \\
F i=3,121\end{array}$ & 5 \\
\hline $\begin{array}{l}\text { Reference \& } \\
\begin{array}{l}\text { User Services } \\
\text { Quarterly }\end{array}\end{array}$ & $\begin{array}{l}\text { Revista oficial de la American } \\
\text { Library Association }\end{array}$ & $\begin{array}{l}\text { American } \\
\text { Library As- } \\
\text { sociation }\end{array}$ & $\begin{array}{l}\text { JCR Q4 } \\
(73 \text { de 88) } \\
\text { Fi=0,377 }\end{array}$ & 4 \\
\hline
\end{tabular}

Tabla 05. Artículos destacados matriz N1.

Elaboración propia con datos de SSCI-WoS

Entre las revistas incluidas en el SSCI es la española El profesional de la información la que más artículos ha publicado sobre las noticias falsas, debido a un monográfico que vio la luz en 2018. Cabe señalar igualmente que un mismo editor (Taylor \& Francis) aglutina 3 de las revistas que más artículos han publicado sobre fake news. Esta editorial publica varias revistas especializadas en periodismo, otro dato que vuelve a situar la investigación sobre este fenómeno en el campo de la comunicación.

También es interesante observar que dos de las revistas que aparecen en este listado están especializadas en Biblioteconomía y Documentación, dato que es consistente con las áreas temáticas más relacionadas con las fake news, según expusimos en el punto anterior.

Lo mismo cabe decir de la presencia de una revista especializada en Psicología experimental, campo que también apareció en la clasificación temática comentada párrafos atrás.

En el caso de la matriz N2, las revistas latinoamericanas que han publicado artículos sobre las fake news son (por orden descendente de artículos):

\begin{tabular}{|l|l|l|c|c|}
\hline \multicolumn{1}{|c|}{ Nombre de la revista } & \multicolumn{1}{|c|}{$\begin{array}{c}\text { Campo } \\
\text { temático }\end{array}$} & \multicolumn{1}{c|}{ País } & ICDS* & $\begin{array}{c}\text { N. }{ }^{\circ} \text { de } \\
\text { artículos }\end{array}$ \\
\hline $\begin{array}{l}\text { Revista Latina de Comunicación } \\
\text { Social }\end{array}$ & $\begin{array}{l}\text { Ciencias Sociales. } \\
\text { Comunicación }\end{array}$ & España & 9,8 & 3 \\
\hline Doxa Comunicación & $\begin{array}{l}\text { Ciencias Sociales. } \\
\text { Comunicación }\end{array}$ & España & 9,7 & 2 \\
\hline $\begin{array}{l}\text { Revista Mediterránea de } \\
\text { Comunicación }\end{array}$ & $\begin{array}{l}\text { Ciencias Sociales. } \\
\text { Comunicación }\end{array}$ & España & 7,5 & 1 \\
\hline Teoría y Realidad Constitucional & $\begin{array}{l}\text { Ciencias Sociales. } \\
\text { Derecho }\end{array}$ & España & 9,8 & 1 \\
\hline Comunicación y Sociedad & $\begin{array}{l}\text { Ciencias Sociales. } \\
\text { Comunicación }\end{array}$ & México & 10 & 1 \\
\hline
\end{tabular}

Tabla 06. Revistas latinoamericanas.

Elaboración propia con datos de Dianet Plus

*Índice Compuesto de Difusión Secundaria obtenido de la Matriz de Información para las

Revistas Científicas (MIAR, 2019) 


\subsection{Correlación temática entre descriptores clave}

Como se ha explicado en la metodología, el corpus de 164 artículos se ha obtenido a raíz de una búsqueda selectiva del descriptor clave de la investigación (fake news) con la etiqueta temática (TS) y el descriptor entrecomillado (TS= "fake news"). Este criterio permite localizar el descriptor exacto en el título, abscract y/o keywords de los artículos contenidos en la base de datos SSCI de la Web of Science, con un resultado de 164 ítems.

Una búsqueda sistemática dentro de ese corpus alternando "fake news" con determinadas palabras clave a través del buscador booleano "AND" nos arroja algunos resultados interesantes para el objetivo de esta investigación.

\begin{tabular}{|c|c|c|}
\hline $\begin{array}{c}\text { ETIQUETA DE CAMPO } \\
\text { TEMÁTICO }\end{array}$ & $\begin{array}{c}\text { BUSCADOR } \\
\text { BOOLEANO }\end{array}$ & N. ${ }^{\text {o }}$ artículos \\
\hline TS= "fake news" & -- & $164(100 \%)$ \\
\hline TS= "media" & AND & $112(68 \%)$ \\
\hline TS= "social media" & AND & $57(34,7 \%)$ \\
\hline TS= "journal*" & AND & $37(22,5 \%)$ \\
\hline TS= "misinformation" & AND & $35(21,3 \%)$ \\
\hline TS= "post-truth" & AND & $26(15,8 \%)$ \\
\hline TS= "disinformation" & AND & $15(9,1 \%)$ \\
\hline TS= "fact-check*" & AND & $15(9,1 \%)$ \\
\hline TS= "verification" & AND & $10(6 \%)$ \\
\hline TS= "media literacy" & AND & $9(5,4 \%$ \\
\hline TS= "false news" & AND & $6(3,6 \%)$ \\
\hline
\end{tabular}

Tabla 07. Principales buscadores booleanos aplicados.

Elaboración propia con datos de SSCI-WoS

De esta forma, al combinar "fake news" con "media" obtenemos una muestra de 112 artículos, lo que indica que el 68\% del corpus vincula el fenómeno de las fake news con los medios de comunicación social. El porcentaje es del 34,7\% cuando la combinación se efectúa con "social media" y del $22,5 \%$ cuando se combina con "journal". La preponderancia de artículos que relacionan el fenómeno de las fake news con los medios de comunicación social y el periodismo es suficientemente llamativa como para tenerla en cuenta en una revisión bibliográfica.

Igualmente, al cruzar "fake news" con las palabras clave "misinformation" $(21,3 \%)$ o "disinformation" $(9,1 \%)$, encontramos interesantes porcentajes de coincidencia. También es resaltable el número de investigaciones que correlacionan "fake news" con "post-truth", con un total de 26 artículos $(15,8 \%)$.

Otros resultados obtenidos permiten localizar 15 artículos con la combinación "fake news" y "fact-check"; 10 con "verification", 9 con "media literacy" y 6 con "false news". 


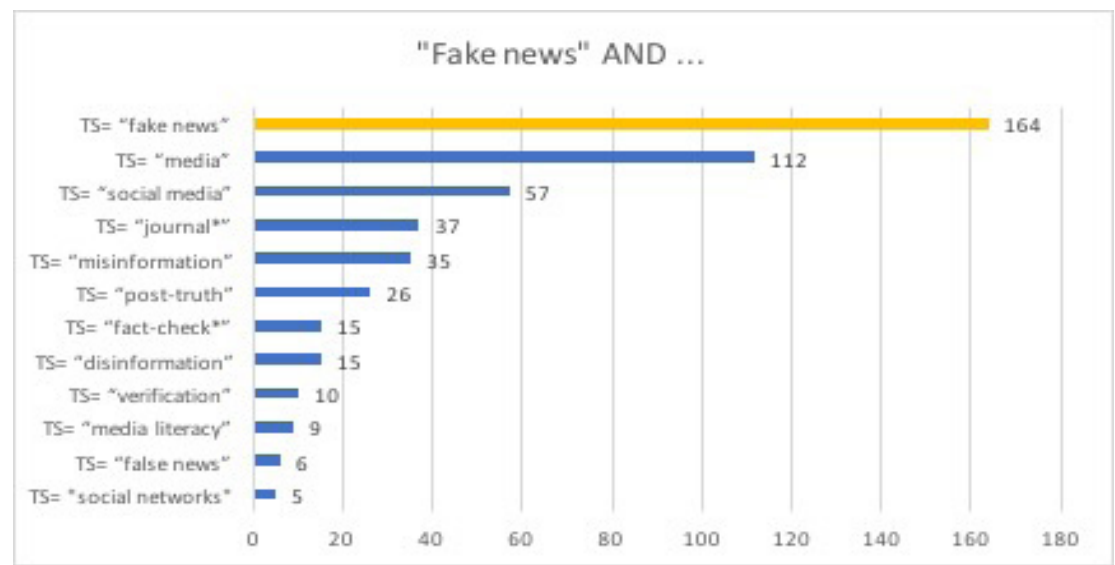

Gráfico 6. Combinaciones de búsqueda con el concepto "fake news".

Elaboración propia con datos de SSCI-WoS

\subsection{Técnicas de investigación}

Como se indicó en la metodología, en este trabajo se ha realizado también un análisis para tratar de identificar y cuantificar las técnicas de investigación empleadas en los artículos que integran el corpus, pues con ello se ofrece un panorama de los recursos metodológicos empleados con mayor profusión para investigar el fenómeno de las fake news.

Para ello, se han identificado y codificado con un sistema de presente (1) / ausente (0) las diversas técnicas de cada ítem analizado, asumiendo que en el campo de las Ciencias Sociales es frecuente la triangulación de métodos y, por lo tanto, que un mismo artículo puede presentar resultados obtenidos con diferentes metodologías.

La identificación de la metodología implica cierta complejidad pues, aun tratándose de artículos publicados en revistas con elevados estándares de calidad científica, en numerosas ocasiones los autores no explican con claridad la metodología o no la identifican con términos normalizados, lo que ha implicado un trabajo ulterior de discernimiento del método empleado.

En la matriz de análisis N1, encontramos 23 artículos (14\%), cuya metodología no aparece especificada, y $32(19,5 \%)$ que consisten en ensayos o aproximaciones teóricas al fenómeno.

Sin embargo, como se observa en el gráfico 7, las técnicas de investigación más empleadas han sido, por este orden, el análisis de contenido (cuantitativo y cualitativo) y el estudio de caso, con 36 artículos identificados en cada caso (un 21,9\% del total, respectivamente). Le siguen los denominados Social Network Analysis con 32 artículos (un 19,5\%) que, en definitiva, trataban de cuantificar la presencia en redes sociales de las noticias falsas. Los artículos basados en revisiones bibliográficas o estados del arte suman $22(13,4 \%)$, seguidos de las encuestas $(9,1 \%)$, los experimentos $(7,9 \%)$, las entrevistas $(6,7 \%)$ y los grupos de discusión o focus group $(2,4 \%)$. 


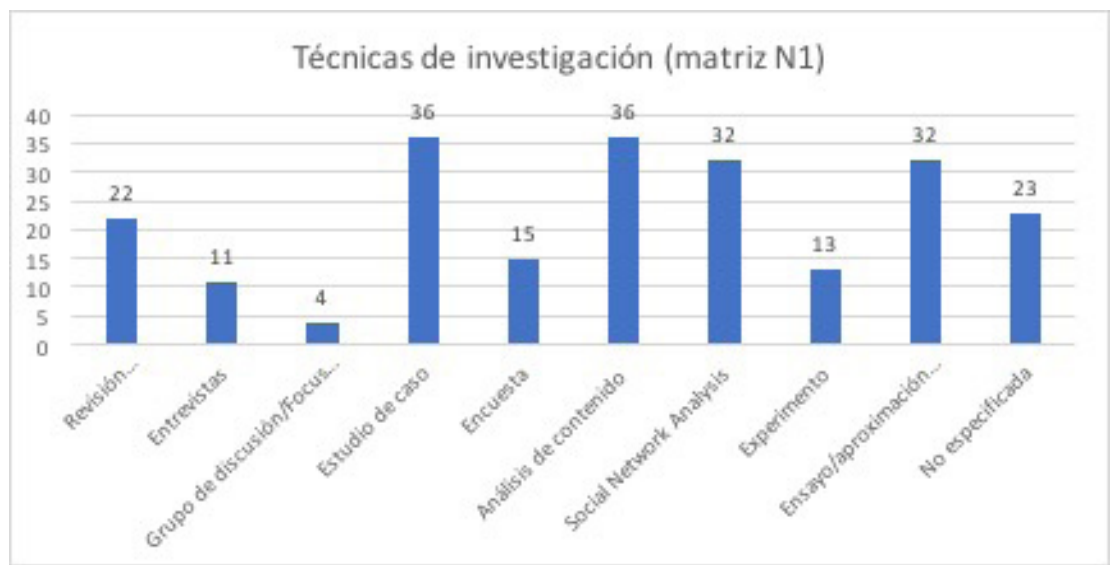

Gráfico 7. Principales técnicas de investigación empleadas (matriz N1).

Elaboración propia con datos de la $W o S$

Entre los ejemplos más representativos de las técnicas de investigación empleadas en estudios sobre las noticias falsas, se podría empezar por destacar el diseño y aplicación de experimentos, presente en 13 artículos de la matriz N1, y que se observa en trabajos como "Advice-taking as an unobtrusive measure of prejudici"; o en el estudio "Fake images: The effects of source, intermediary, and digital media literacy on contextual assessment of image credibility online", que desarrolla un experimento en línea para investigar cómo las personas evalúan la credibilidad de la imagen en las plataformas en línea. Esta técnica aparece igualmente en otros títulos, como "Fighting misinformation on social media using crowdsourced judgments of news source quality" o "A corpus of debunked and verified user-generated videos", que aplica un conjunto de experimentos de verificación automática a partir de información derivada de 380 videos generados por el usuario (UGV) junto con 5.195 versiones de ellos.

Por otra parte, el uso de las entrevistas está presente en 11 de los artículos analizados. Son trabajos como "Young people's conceptions of political information Insights into information experiences and implications for intervention", que utilizó está técnica para explorar las formas en que 23 jóvenes de 14 a 15 años evaluaban la calidad de la información. En la misma línea, la entrevista en profundidad aparece en artículos como "Fake news Australian and British journalists' role perceptions in an era of 'alternative facts", que entrevista a periodistas de alto nivel con sede en Londres y Sydney para determinar cómo entienden su papel en una era de noticias falsas.

El análisis de contenido es la técnica en la que se han basado 36 artículos, como "Influence politics and "fake" trend on Twitter. $21 D$ post-election effects within the Proces in Catalonia", donde a partir de una metodología triangular de análisis de contenido, se analizan las principales estrategias y los posibles efectos de una comunicación digital, en la que el sujeto (quién) prevalece antes del objeto (qué). En este campo, destaca, además, entre otros estudios, el artículo "Prior Exposure Increases Perceived Accuracy of Fake news", que analiza los titulares de noticias falsas de Facebook.

La altísima viralización de noticias falsas a través de las redes sociales convierte al Social Network Analysis en una técnica recurrente para investigar este fenómeno. Está presente en 32 artículos del corpus N1, como "The diffusion of misinformation on social media: Temporal pattern, message, and source", que rastrea el ciclo de 
vida de 17 rumores políticos populares que circularon en Twitter durante 13 meses aplicando el análisis de texto con diferentes parámetros. "Serbia in the Mirror: Parodying Political and Media Discourses" es un artículo que analiza el sitio de noticias falsas serbio Njuz.net, explorando las dinámicas de su producción, consumo y apropiación en la etapa previa a la adhesión a la UE. El trabajo "The small, disloyal fake news audience: The role of audience availability in fake news consumption" destaca por presentar un estudio realista del ecosistema digital.

Por otro lado, trabajos como "FakeNewsTracker: a tool for fake news collection, detection, and visualization" se centran, desde la lógica del estudio de caso, en el análisis de experiencias concretas como, en este caso, FakeNewsTracker, un sistema para la comprensión y detección de noticias falsas. También se detecta esta técnica en el estudio "Analyzing the Social Construction of Media Claims: Enhancing Media Literacy in Social Problems Classes", que describe un proyecto de alfabetización mediática orientado a que los alumnos sean capaces de ver cómo los medios comunican sobre determinados problemas sociales.

Finalmente, se encontró únicamente un artículo que presenta una revisión de cómo estudios previos han definido el término "fake news". Bajo el título de "Defining Fake news: A typology of scholarly definitions", el trabajo analiza 34 artículos académicos que utilizaron esta expresión entre 2003 y 2017.

En el caso de la matriz N2, las técnicas de investigación identificadas están más concentradas debido a un corpus menos numeroso. No obstante, se observa la preferencia por las metodologías cualitativas ya detectada en la matriz N1.

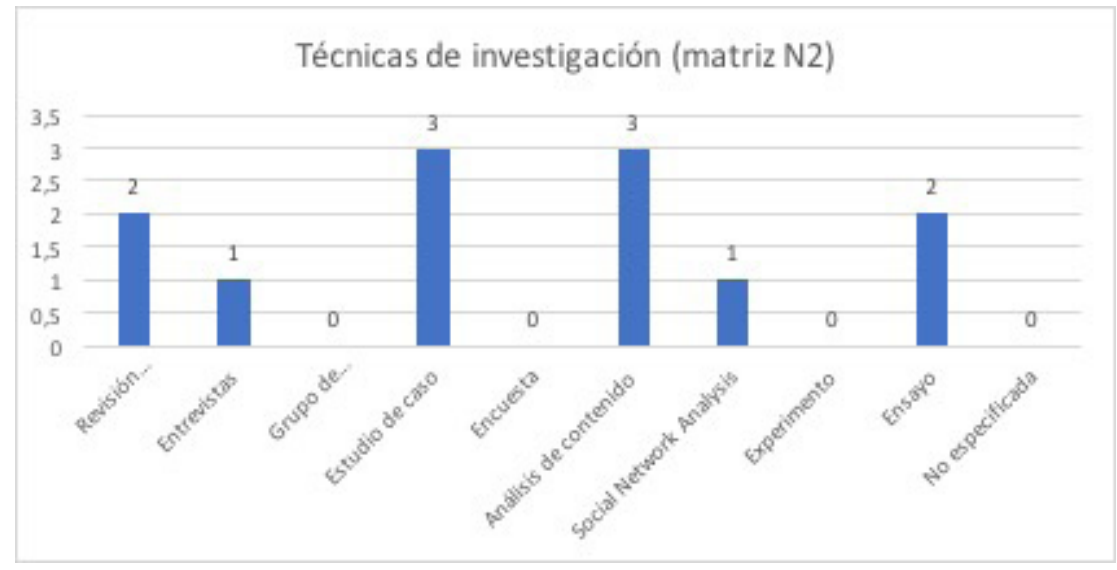

Gráfico 8. Principales técnicas de investigación empleadas (matriz N2).

Elaboración propia con datos de Dialnet Plus

El análisis de contenido está presente en tres de los trabajos publicados: "La información digital enjuiciada por la prensa", "Fact-Checking en los debates electorales televisados de las elecciones generales de 2015 y 2016", y "WhatsApp como herramienta de verificación de fake news. El caso de B de Bulo". Este último también emplea la técnica del estudio de caso, presente además en estos dos títulos: "El rumor del nopal chino: construcción institucional y efectos sociales de noticias falsas" y "El Slow Journalism en la era de la "infoxicación". Al igual que el análisis de contenido, son 3 los estudios de caso encontrados, con lo que ambas técnicas fueron utilizadas en el $37,5 \%$ de las investigaciones. 
Los artículos que ofrecen estados del arte o revisiones bibliográficas son dos: "Noticias falsas y libertad de expresión e información. El control de los contenidos informativos en la red” y "¿Cómo afrontar las noticias falseadas mediante la alfabetización periodística? Estado de la cuestión"; al igual que los que proponen una aproximación teórica o ensayística, como es el caso de "Estratagemas de la posverdad". Por último, la entrevista y el análisis de las redes sociales serán las técnicas de investigación empleadas en los dos artículos restantes.

\section{Conclusiones}

A partir del conjunto de resultados derivados de la presente revisión sistematizada de la literatura científica que existe con relación al fenómeno de las fake news en el ámbito de las Ciencias Sociales es posible articular un conjunto de reflexiones a modo de conclusiones.

En primer lugar, los resultados obtenidos permiten validar la hipótesis de la investigación que señalaba que la principal producción científica sobre fake news procede del ámbito científico anglosajón. En la misma línea, el estudio ha permitido verificar que este conjunto de trabajos se centra en el ámbito de la Comunicación y que su eclosión se ha producido en los dos últimos años a raíz, especialmente, de los acontecimientos políticos que tuvieron lugar en el año 2016. De este modo, es posible concluir que las campañas políticas del referéndum del Brexit (junio 2016) y de las elecciones presidenciales en Estados Unidos (noviembre 2016) constituyen un punto de inflexión en la producción científica sobre este tema que, desde ese momento, experimenta un llamativo crecimiento.

Por otro lado, los resultados obtenidos permiten identificar las noticias falsas como un ámbito temático de gran impacto entre la comunidad científica de Ciencias Sociales, tanto a nivel iberoamericano como en el escenario anglosajón. En este sentido, tanto la Web of Science como Scopus muestran una estrecha coincidencia respecto al conjunto de artículos resultantes. Con relación a ello, se observa que la denominación de "fake news" suele vincularse con otros conceptos o descriptores, tales como "misinformation", "disinformation", "news verification", "false news" y "fact checking". Este aspecto refuerza los planteamientos de autores como Wardle y Derakhshan (2017) que han señalado la importancia de desarrollar estudios que enmarcan de forma más elocuente el alcance del término y la globalidad de la problemática que le rodea.

La distribución de artículos por países (según la procedencia de su autoría) denota la necesidad de potenciar la producción científica a nivel global. Entre los países que mayor número de trabajos han elaborado sobre este campo, sobresale Estados Unidos (con 79 artículos, cifra que supone cerca de un 50\% del total), cuyos datos doblan al resto de países juntos (que suman 85 artículos). Junto al incremento del volumen de publicaciones, resulta igualmente pertinente el desarrollo de proyectos conjuntos entre países y/o universidades en aras de abordar de forma más rica y completa un fenómeno que posee un talante claramente global.

A nivel temático, la Comunicación, junto a la Biblioteconomía, son el escenario con mayor presencia de este tipo de trabajos. Este aspecto, que podría justificarse debido a que es en el ámbito de las Ciencias Sociales donde existe más preocupación sobre este fenómeno, advierte de la necesidad de impulsar trabajos interdisciplinares 
que permitan la implicación de investigadores de diferentes áreas o campos de gran interés y provecho para el abordaje teórico y práctico del objeto de estudio de las fake news (como la Psicología, la Educación, el Derecho, la Ingeniería o la Sociología, entre otros). Se detecta, en definitiva, una carencia de propuestas interdisciplinares que potencien la hibridación de saberes en los enfoques teóricos y en el diseño de los engranajes metodológicos para el estudio de esta problemática. En esta línea, se observa la necesidad de diversificar las miradas hacia este objeto de estudio con el objetivo de superar el escenario de los medios de comunicación social y del periodismo para introducir otras facetas donde serían de gran valor estudios relacionados con las fake news, como la educación, la ingeniería o el derecho, entre otros.

En el plano de la metodología empleada, el estudio permite concluir que existe un predominio de ensayos o aproximaciones teóricas al fenómeno, al tiempo que se observa una preeminencia de técnicas como el análisis de contenido (cuantitativo y cualitativo), el estudio de caso y los Social Network Analysis. Existe, no obstante, un número muy reducido de trabajos centrados en el diseño y aplicación de experimentos, así como en la realización de grupos de discusión.

Finalmente, las fake news pueden concebirse como un escenario proclive al desarrollo de trabajos científicos por parte de los docentes, investigadores y grupos de investigación del ámbito iberoamericano, que se encuentra muy alejado del volumen de investigaciones desarrolladas por la academia anglosajona. A ello se une la importancia de que las revistas científicas iberoamericanas potencien los monográficos $\mathrm{o}$, en definitiva, la presencia de esta temática en sus respectivos números.

\section{Referencias bibliográficas}

Blanco, I. (2011). "Libertad y prensa, de Walter Lippmann". En: Revista Doxa Comunicación, $\mathrm{n}^{\circ}$ 13, Madrid: CEU San Pablo, pp. 241-244.

Botella, J.; Gambara, H. (2006). El meta-análisis: una metodología de nuestro tiempo. Infocop. Recuperado de http://www.infocop.es/view_article.asp?id=843

Camili, C. (2018). "La educación mediática en España: revisión sistemática de la producción académica publicada en revistas científicas". En Fuente, García y Camili (coord.) (2018). La educación mediática en España. Artículos seleccionados. Madrid: Universitas Editorial. pp. 15-44.

Codina, L. (2018). Revisiones sistematizadas para trabajos académicos 1: Conceptos, fases y bibliografía. Recuperado de https://www.lluiscodina.com/revisiones-sistematizadas-fundamentos/

- (2017). Investigación con bases de datos. Estructura y Funciones de las Bases de Datos Académicas Análisis de Componentes y Estudio de Caso. Materiales para el Máster Universitario en Comunicación Social (MUCS). Recuperado en https://repositori.upf.edu/ bitstream/handle/10230/28135/Codina_2017_estrucbd.pdf?sequence=1\&isAllowed=y

Con licencia, 31-03-2017. Los bots superan a los humanos en presencia web. Recuperado de http://blogconlicencia.com/los-bots-superan-a-los-humanos-en-presencia-web/

Eldiario.es, 23-04-2019. Las mentiras pronunciadas por los candidatos en el debate electoral de RTVE. Recuperado en https://www.eldiario.es/eldetectordementiras/mentiras-pronunciadas-candidatos-electoral-RTVE_0_891710875.html

Flecther, R.; Cornia, A.; Graves, L.; Rasmus, K. (2018). Measuring the reach of fake news and online disinformation in Europe. Oxford: Reuters Institute, University of Oxford. 
Ny Books, 13-12-2016. The Putin Paradigm. Recuperado en https:/www.nybooks.com/daily/2016/12/13/putin-paradigm-how-trump-will-rule/

Guirao, G.; Silamani, J. (2015). "Utilidad y tipos de revisión de literatura". En: Revista de Enfermería, $n^{\circ}$ 9, Canarias: ENE.

Hargittai, E.; Fullertom, L.; Menchen-Trevino, E.; Thomas, K. (2010). “Trust online: Young adults' evaluation of web content". En: International Journal of Communication, California: USC Annenberg Press, nº 4, pp.468-494.

Ireland, S. (2018). "Fake news alerts: teaching news literacy skills in a meme world". En: The Reference Librarian, $\mathrm{n}^{\circ}$ 59, Londres: Taylor and Francis Group, pp. 122-128.

Jang, S.; Mo, J. K. (2018). "Third person effects of fakenews: Fakenews regulation and media literacy interventions". En: Computers in human Behavior, $\mathrm{n}^{\circ}$ 80, Amsterdam: Elsevier, pp. 295-302.

La Vanguardia, 21-02-2019. Las “fake news”, una amenaza para la democracia. Recuperado de https://www.lavanguardia.com/politica/20190221/46612593970/las fake-news-una-amenaza-para-la-democracia.html

López-García, X.; Silva-Rodríguez, A.; Vizoso-García, A.; Westlund, O.; Canavilhas, J. (2019). "Mobile journalism: Systematic literature review". En: Comunicar, no 59, Huelva: Grupo Comunicar, pp. 9-18.

Love, R. (2007). "Before Jon Stewart". En: Columbia Journalism Review, n 45, Nueva York: Columbia University, pp. 33-37.

Lowrey, W. (2017). "The Emergence and Development of News Fact-checking Sites". En: Journalism Studies, n ${ }^{\circ}$ 18, Londres: Taylor and Francis Group, pp. 376-394.

Middaugh, E. (2019). "More Than Just Facts: Promoting Civic Media Literacy in the Era of Outrage”. En: Peabody Journal of Education, $n^{\circ}$ 94, Londres: Taylor and Francis Group, pp. 17-31.

Musgrove, A.; Powers, J.; Rebar, L.; Musgrove, G. (2018). "Real o fake? Resources for teaching college students how to identify fake news". En: Collegue \& Undergraduate Libraries, $\mathrm{n}^{\circ} 25$, Londres: Taylor and Francis Group, pp. 243-260.

Peña, M. (2012). "La importancia del acuerdo entre codificadores para el análisis de contenido". En: Comunicación y Medios, $\mathrm{n}^{\circ} 25$, Santiago de Chile: Universidad de Chile, pp. 47-56.

Pérez Tornero, J.m.; Tayie, S.; Tejedor, S.; Pulido, C. (2018). “Cómo afrontar las noticias falseadas mediante la alfabetización periodística? Estado de la cuestión". En: Doxa Comunicación, $\mathrm{n}^{\circ}$ 26, Madrid: CEU San Pablo, pp. 211-235.

Pérez-Tornero, J. M.; Varis, T. (2010). Media Literacy and New Humanism. París: UNESCO. RTVE (2019). El equipo de verificación de RTVE desvela las afirmaciones falsas de los candidatos durante el debate. Recuperado en http://www.rtve.es/temas/debate-en-rtve/125810/

Sánchez-Aranda, J. J. (2005). “Análisis de contenido cuantitativo de medios”. En Berganza, R.; Ruiz, J. A. (ed.) (2005). Investigar en comunicación: guía práctica de métodos y técnicas de investigación social en comunicación. Barcelona: McGraw Hill. pp.207-228.

Southwell, B.; Thorson, E.; Sheble, L. (eds.) (2018). Misinformation and mass audiencies. Austin: University of Texas.

Tally, R. (2011). "I am the mainstream media (and so can you!)". En AMARASINGAM, A. (ed.) (2011). The Stewart/Colbert effect. Essays on the real impacts offake news. Carolina del Norte: McFarland. pp. 149-163.

Tannen, D. (1999). La cultura de la polémica. Barcelona: Paidós.

Thompson, M. (2016). Enough Said: What's Gone Wrong with the Language of Politics? London: The Bodley Head. 
Turkle, S. (2015). Reclaiming Conversation: The Power of Talk in a Digital Age. Nueva York: Penguin Press.

Vizoso, A.; Vázquez-Herrero, J. (2019). "Fact-checking platforms in Spanish. Features, organisation and method". En: Communication \& Society, $n^{\circ} 32$, Navarra: Universidad de Navarra, pp. 127-143.

Vosoughi, S.; Roy, D.; Aral, S. (2018). "The Spread of true and false news online”. En: Siencie, $\mathrm{n}^{\mathrm{o}}$ 359, Washington: AAAS, pp. 146-1151.

Warldle, C.; Derakhshan, H. (2017). Information disorder. Toward an interdisciplinary framework for research and policymaking. Estrasburgo: Council of Europe report DGI.

Wilson, V. (2013). "Research Methods: Systematic Reviews". En: Evidence Based Library and Information Practice, $\mathrm{n}^{\circ}$ 8, Edmonton: University of Alberta, pp. 83-84.

Wineburg, S.; Mcgrew, S. (2016). "Why students can't google their way to the truth". En: Education Week, no 36, Bethesda: Editorial Projects in Education, pp. 22-28. 